\title{
Ultra-sonografia na Artrite Reumatóide: Aplicabilidade e Perspectivas
}

\section{Ultrasonography in Rheumatoid Arthritis: Applicability and Expectations}

\author{
Eloy de Ávila Fernandes ${ }^{(1)}$, Moacir Ribeiro de Castro Junior ${ }^{(2)}$, Sônia de Aguiar Vilela Mitraud ${ }^{(3)}$, \\ Edson Shinji Kubota ${ }^{(4)}$, Artur da Rocha Corrêa Fernandes ${ }^{(5)}$
}

\section{RESUMO}

Este trabalho realiza uma revisão bibliográfica sobre o papel do ultrasom (US) no diagnóstico precoce da artrite reumatóide (AR), sua utilização no tratamento destes pacientes e sua utilidade na avaliação da resposta ao tratamento. A AR é uma doença inflamatória crônica que, quando não diagnosticada e tratada adequadamente, pode levar à deformidade física e à incapacidade para o trabalho. A introdução precoce das drogas modificadoras da doença (DMCD), sejam elas tradicionais ou mais novas (agentes biológicos), pode modificar a evolução da doença. Para tanto, o diagnóstico da AR precisa ser feito o mais rápido possível. O US é um método que possibilita o estudo das articulações e pode mostrar sinais de atividade inflamatória, especialmente a sinovite. Técnicas como o estudo com Doppler colorido e o Doppler de amplitude podem ajudar na avaliação de atividade da doença, diferenciando tecido inflamatório ativo (pannus) de inativo. Outros achados como erosões, alterações tendíneas, bursites, cistos sinoviais e derrames articulares também podem ser encontrados. Esta revisão, no entanto, demonstra que métodos de quantificação da atividade inflamatória ainda precisam ser estabelecidos no US.

Palavras-chave: artrite reumatóide, ultra-som, sinovite, erosão.

\section{INTRODUÇÃO}

A artrite reumatóide (AR) é uma doença inflamatória crônica, com prevalência estimada em $0,5 \%$ a $1,0 \%$ da população adulta, e as mulheres são duas a três vezes mais acometidas que indivíduos do sexo masculino. A incidência no sexo feminino é de 54 por 100.000 habitantes e no sexo masculino de 25 por 100.000 habitantes. O início geralmente ocorre entre os 30 e 50 anos e $20 \%$ a $30 \%$ dos pacientes não tratados tornam-se incapacitados para o trabalho em dois a três anos do diagnóstico da doença ${ }^{(1,2)}$.

\begin{abstract}
This review discusses the usefulness of ultrasound in the early diagnosis of reumathoid arthritis, its utilization in these patients treatment and its utility in evaluation of the response to treatment. Reumathoid arthritis is a chronic disease that when it is not promptly diagnosed it can cause physical deformity and disability to work. The early introduction of disease modifying antirheumatic drugs (DMARDs), no matter traditional or newer (biologic agents), may modify the disease outcome. In order that, the diagnosis of RA must be made as soon as possible. Ultrasound makes possible to study the joints and can show signs of inflammatory activity, especially synovitis. Color Doppler and power Doppler can help differentiate between active inflammatory tissue (pannus) and inactive inflammatory tissues. Erosions, tenossynovitis, bursitis, cysts and effusions can also be found. This article demonstrates that methods to quantify de inflammatory activity on ultrasound must yet be established.
\end{abstract}

Keywords: rheumatoid arthritis, ultrasound, synovitis, erosion.

A etiologia da AR não é completamente conhecida, atribuindo-se a ela fatores ambientais e genéticos. Como evidências de causas genéticas observam-se em gêmeos monozigóticos até mais que 30\% de concordância no desenvolvimento de AR e nota-se que certos alelos HLA-DRI ou DR4 estão presentes em $80 \%$ dos pacientes caucasianos com artrite reumatóide. Fatores ambientais como o tabagismo ou agentes infecciosos podem ter papel importante na etiologia da $\mathrm{AR}$, mas sua contribuição ainda não está definida ${ }^{(1,2,3)}$.

Recebido em 28/8/2007. Aprovado, após revisão, em 17/12/2007. Declaramos a inexistência de conflitos de interesse

Departamento de Diagnóstico por Imagem da Universidade Federal de São Paulo (Unifesp).

1. Médico radiologista, colaborador do Departamento de Diagnóstico por Imagem da Escola Paulista de Medicina da Universidade Federal de São Paulo

(Unifesp/EPM)

2. Médico radiologista, colaborador do Departamento de Diagnóstico por Imagem da Unifesp/EPM.

3. Médica radiologista do Setor de Músculo Esquelético do Departamento de Diagnóstico por Imagem da Unifesp/EPM

4. Médico radiologista do Setor de Músculo Esquelético do Departamento de Diagnóstico por Imagem da Unifesp/EPM

5. Professor doutor adjunto e vice-chefe do Departamento de Diagnóstico por Imagem da Unifesp/EPM.

Endereço para correspondência: Departamento de diagnóstico por imagem, secretaria. Rua Napoleão de Barros, 800, térreo, CEP 04024-002, São Paulo, SP, e-mail:

loferna@uol.com.br 
$\mathrm{Na} A R$, o sítio do processo patológico é a sinóvia, com acometimento das articulações sinoviais e bainhas tendíneas. $\mathrm{O}$ processo inicia-se com a proliferação de macrófagos e fibroblastos sinoviais depois de um fator desencadeante, provavelmente auto-imune ou infeccioso. Observa-se um infiltrado linfocítico nas regiões perivasculares e proliferação de células, com conseqüente neovascularização, hiperplasia sinovial e formação de pannus. $\mathrm{Na}$ articulação sinovial, a superfície dos osssos que se articulam é coberta por cartilagem, exceto uma pequena região entre a inserção da cápsula fibrosa e a cartilagem, onde o osso é coberto somente por sinóvia, a chamada área nua da articulação. Em contato direto com o tecido sinovial, sem qualquer camada protetora de cartilagem, a superfície óssea nesse local é muito suscetível à destruição óssea induzida por sinovite ${ }^{(1,4,5)}$. A proliferação do tecido sinovial nesse local é um achado precoce. Angiogênese e hipervascularização e conseqüente formação de pannus constituem um mecanismo patogênico essencial, que leva à destruição articular, cartilagínea e óssea, durante a progressão da AR. A presença de vascularização no pannus pode distinguir o processo inativo do inflamatório, tendo impacto importante na conduta terapêutica ${ }^{(4)}$. A diferenciação entre tecido articular sinovial fibrótico inativo de pannus (ativo) e a quantificação da sinovite é, atualmente, importante área de investigação ${ }^{(6)}$.

Substâncias como citocinas (interleucina 1 ), fatores de crescimento e proteinases também participam da destruição das estruturas articulares e periarticulares, bem como do desenvolvimento de manifestações sistêmicas ${ }^{(25,7)}$.

A ultra-sonografia pode ser amplamente utilizada na prática diária em reumatologia, permitindo o estudo de partes moles e ósseas, apresentando as seguintes vantagens: é um método não-invasivo, de menor custo comparado à ressonância magnética, aplicável a diversas articulações e possibilita o estudo dinâmico destas. Além disso, tem-se o uso do Doppler colorido e do Doppler de amplitude, este último melhor para a avaliação de fluxo de baixa velocidade em estruturas que contenham pequenos vasos como a sinóvia ${ }^{(6,8,9)}$.

Entretanto o ultra-som é um método operador-dependente, exigindo profissionais com grande experiência no método, conhecimento de anatomia, de patologias musculoesqueléticas e com capacidade de reconhecer artefatos, os quais muitas vezes podem simular lesões. Outro fator determinante é a sofisticação técnica do equipamento, que influencia na resolução da imagem e conseqüentemente no diagnóstico, além de o fato de transdutores de baixa freqüência, menores que $7,5 \mathrm{MHz}$, não serem apropriados para o acesso detalhado de pequenas estruturas anatômicas superficiais ${ }^{(10,11)}$. Os transdutores de baixa freqüência como de 3,5 e $5 \mathrm{MHz}$ servem para analisar estruturas profundas, já os transdutores com freqüência de 7,5 MHz ou mais são utilizados para estudo de estruturas superficiais, visto que quanto maior a freqüência do transdutor menor a penetração do feixe sonoro e melhor a resolução espacial.

\section{ACHADOS ULTRA-SONOGRÁFICOS}

\section{SINOVITE}

A sinovite é o achado mais precoce identificado ao ultra-som e é definida como uma área hipoecóica, não deslocável e pouco compressível, intracapsular e distinta da gordura intra-articular que se apresenta hiperecóica. A sinóvia normal não é identificada ao US ${ }^{(10-13)}$. Para a avaliação de sinovite na ultra-sonografia, estudos têm sido propostos para quantificar o espessamento sinovial, como o de Szkudlarek et al. ${ }^{(14)}$, em 2004, que utilizou medidas semiquantitativas volumétricas, sugerindo que as alterações inflamatórias são similares às medidas obtidas por intermédio da ressonância magnética ${ }^{(11,14,15)}$.

Ostergaard et al. ${ }^{(15)}$ propuseram uma modificação do sistema de graduação previamente descrito em articulações metatarso-falângicas no plano longitudinal, classificando o grau de espessamento sinovial em cinco estágios. No grau zero, não há espessamento sinovial, no grau 1 há mínimo espessamento sinovial, no grau 2 há espessamento sinovial abaulando sobre a linha que liga as porções mais altas dos ossos que formam a articulação, sem extensão para as diáfises, no grau 3 há o mesmo que no grau 2 , com extensão para uma das diáfises e no grau 4 há o mesmo que no grau 2 , com extensão para ambas as diáfises ${ }^{(15)}$ (Figura 1 ).

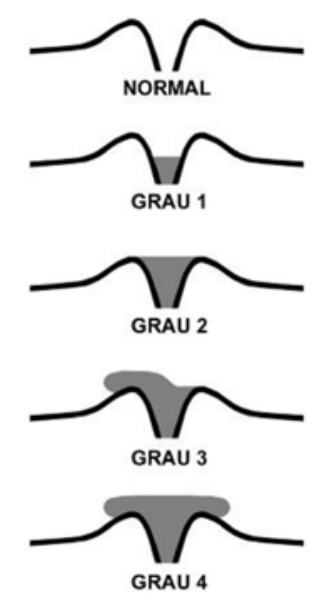

Figura 1 - Esquema de articulação metacarpo-falângica exemplificando os cinco graus anteriormente descritos por 0stergaard et $a l^{15}$. 
Farrant et al. ${ }^{(11)}$ referem que esses estudos devem ser tratados com cautela porque poucos pacientes foram envolvidos neles e havia apenas um único observador, havendo falta de concordância intra-observador.

A sensibilidade e a especificidade do ultra-som para detecção de sinovite são comparáveis à ressonância magnética nas pequenas articulações de mãos, pés e grandes articulações e superiores ao exame clínico e ao estudo radiológico ${ }^{(16-20)}$. Hermann et al. ${ }^{(21)}$, em 2003, relataram que a sensibilidade da ressonância magnética na detecção de sinovite e erosões é maior na ressonância magnética que no ultra-som, porém ambos os métodos têm sensibilidade maior que o estudo radiográfico, e sugerem que o ultra-som e a ressonância magnética devam ser utilizados na avaliação diagnóstica inicial da AR quando a radiografia obtiver resultados negativos. Esse estudo apresenta pequeno número de pacientes e apenas um observador realizou o exame ultra-sonográfico, porém confirma o fato da maior sensibilidade da ultra-sonografia e da ressonância magnética em relação ao estudo radiológico.

A OMERACT (The Outcome Measures In Rheumatoid Arthritis Clinical Trials) publicou em 2007 uma revisão sistemática do ultra-som para a detecção de sinovite na artropatia inflamatória. O trabalho considerou características de reprodutibilidade, acurácia e sensibilidade do ultra-som em artigos publicados no PubMed de janeiro de 1966 a junho de 2005, limitados ao idioma inglês. Os autores encontraram pouca informação na literatura comparando ultra-sonografia com achados histopatológicos ou cirúrgicos macroscópicos. Houve pouca padronização na técnica para avaliação de pequenas articulações, o que prejudica a reprodutibilidade do método. Esse foi um fator importante de deficiência nos trabalhos, sendo portanto necessários estudos prospectivos para avaliação da reprodutibilidade ${ }^{(22)}$.

O estudo com Doppler pode detectar fluxo sanguíneo na sinovite. O Doppler colorido mostra a direção e a taxa de fluxo sanguíneo circulante e relaciona-se à velocidade e à direção das células sanguíneas, entretanto sua baixa relação sinal-ruído pode prejudicar a avaliação de vasos profundos ou que contenham fluxo sanguíneo lento. O Doppler de amplitude (power Doppler) mostra a amplitude do sinal de Doppler que é determinado pelo volume de sangue presente. Portanto, o Doppler colorido é melhor na avaliação de fluxos de alta velocidade em grandes vasos, por exemplo, as carótidas, enquanto o Doppler de amplitude (power Doppler) é melhor para acessar fluxos de baixa velocidade e, desse modo, oferece mais informações nas doenças osteoarticulares $^{(9,23)}$. O Doppler de amplitude também é mais sensível a artefatos de movimento, não indica a direção do fluxo e está associado à atenuação quando aumenta a profundidade da estrutura estudada ${ }^{(24)}$.

Imagens obtidas por ressonância magnética e Doppler de amplitude correlacionam a vascularização do tecido sinovial $^{(8,9,25,26)}$, sendo observado fluxo lento ao Doppler na atividade inflamatória sinovial ${ }^{(26)}$. Trabalhos têm comparado favoravelmente o Doppler de amplitude à atividade inflamatória da doença ${ }^{(27)}$ e monitoramento de tratamento, em comparação à tradicional escala de cinza do ultra-som; no entanto, mais estudos serão necessários nessa área ${ }^{(28)}$.

O uso de agentes de contraste intravenoso geralmente otimiza as informações de Doppler ${ }^{(29)}$, porém o alto custo e o caráter invasivo limitam seu uso rotineiro. A proliferação sinovial é facilmente reconhecida sem agente de contraste ultra-sonográfico e não existe ainda consenso quanto sua utilização, em razão de estudos com novas substâncias e do surgimento de equipamentos de ultra-som com melhor sensibilidade na detecção de fluxo ${ }^{(10,18)}$ (Figura 2).

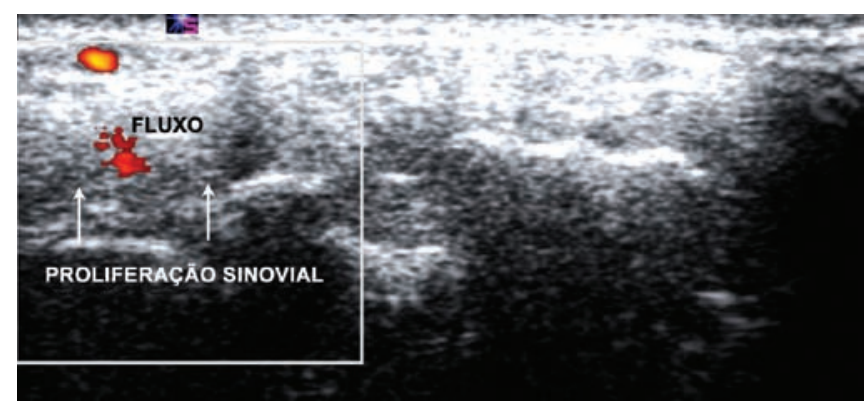

Figura 2 - Pannus. Corte longitudinal do punho na região dorsal evidenciando proliferação sinovial (setas) com fluxo ao power Doppler. Posteriormente observam-se as corticais dos ossos do carpo.

\section{EROSÕES}

Segundo OMERACT, erosão é uma descontinuidade da superfície óssea intra-articular visível em dois planos perpendiculares ${ }^{(30)}$. Erosões observadas ao ultra-som ocorrem com freqüência em articulações assintomáticas (Figura 3). Nas articulações metacarpo-falângicas, as erosões podem ser mais proeminentes na região radiovolar das cabeças dos metacarpos, assim como nos metatarsos ${ }^{(31)}$. Exames de ultra-som dos pés revelaram alterações erosivas em pacientes que tiveram achados ultra-sonográficos negativos nas mãos, sendo mais freqüentemente encontradas erosões no quinto e primeiro metatarso ${ }^{(31)}$. 


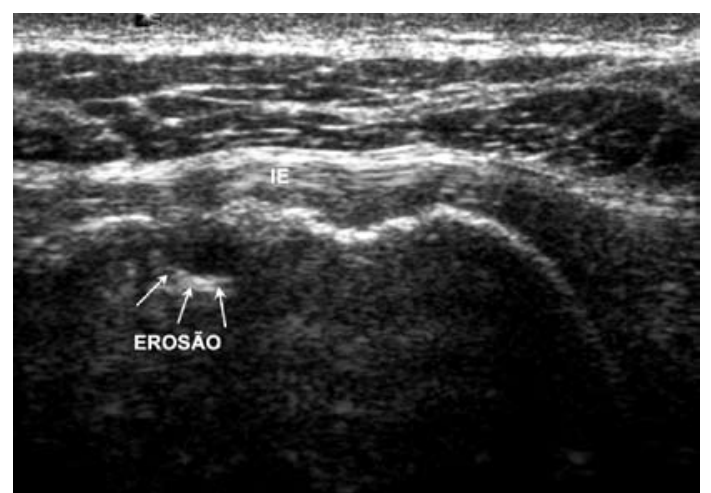

Figura 3 - Erosão óssea. Corte transversal da região posterior da cabeça umeral mostrando erosão óssea em paciente com artrite reumatóide. Anteriormente observa-se o tendão do músculo infra-espinal (IE).

O ultra-som deve ser utilizado na pesquisa de erosões ósseas quando a radiografia for negativa, pela capacidade de detecção de lesões ósseas mais precocemente. A presença de erosões no início dos sintomas pode indicar doença grave $\mathrm{e}$, conseqüentemente, afetar as decisões terapêuticas ${ }^{(6,20)}$ (Figuras 4a e 4b).

A
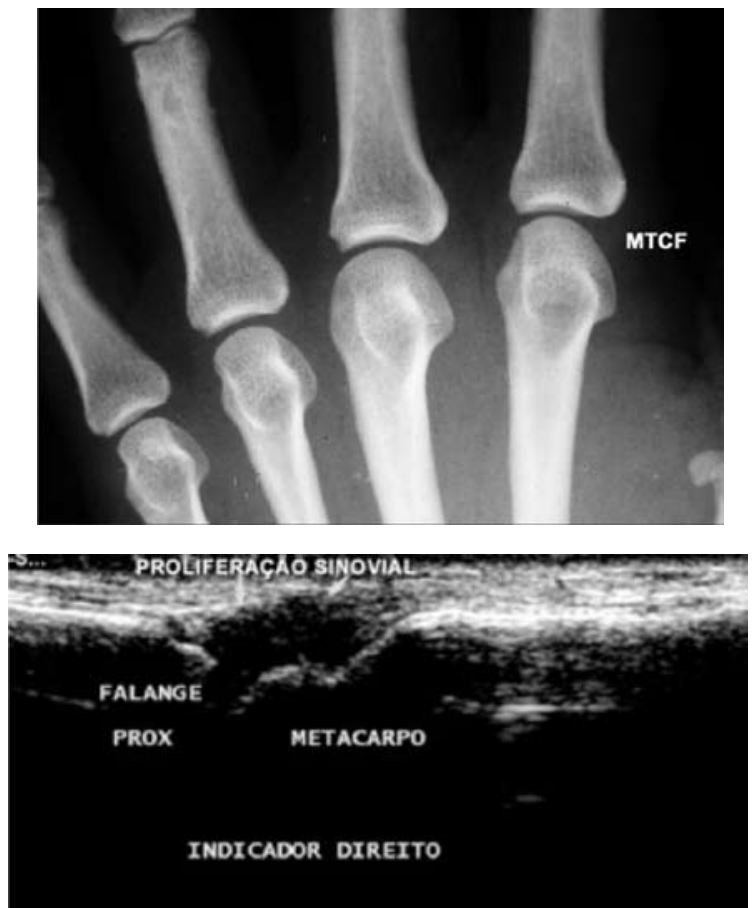

Figura 4 - Paciente portadora de AR. a) Radiografia da mão com discreta redução dos espaços articulares metacarpo-falângicos (MTCF) do segundo, terceiro e quinto quirodáctilos; b) Ultra-som mostrando no corte longitudinal na região dorsal da articulação metacarpo-falângica do segundo quirodáctilo área hipoecóica de proliferação sinovial junto à superfície óssea na área nua da articulação. Anteriormente, observa-se parte do tendão extensor do dedo.

\section{OUTRAS ESTRUTURAS AVALIADAS}

$\mathrm{Na}$ avaliação de tendões, o ultra-som é geralmente melhor que a ressonância magnética e é descrito como método de imagem padrão-ouro para avaliar envolvimento tendíneo na $\mathrm{AR}^{(16)}$. É um bom método para avaliação de tenossinovite $^{(32)}$ (Figura 5). Na avaliação dos tecidos articulares ou periarticulares, podem-se ainda encontrar: sinais de rupturas tendíneas, bursites (Figura 6), cistos sinoviais, coleções intra e extracapsulares e entesites. Derrame articular também pode ser observado precocemente na artrite reumatóide e é facilmente observável na ultra-sonografia como uma área anecóica compressível intracapsular ${ }^{(13)}$ A cartilagem pode ser visualizada pelo ultra-som, mas muitas superfícies articulares são inacessíveis ao método e ainda não foram realizados estudos sistemáticos para avaliar cartilagem na $\mathrm{AR}^{(15)}$

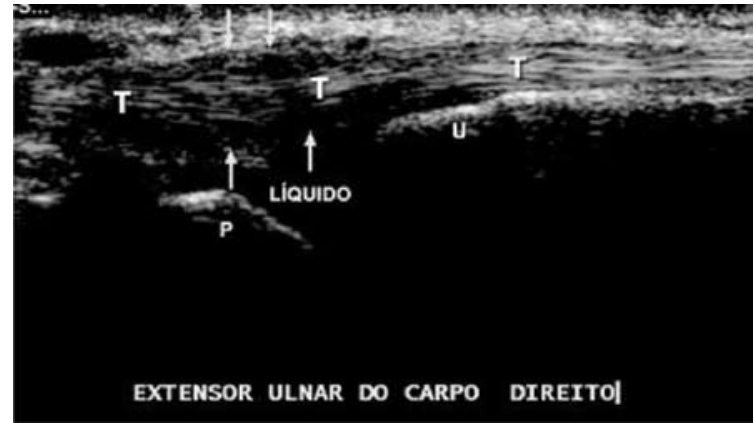

Figura 5 - Tenossinovite do extensor ulnar do carpo. Corte longitudinal no sexto compartimento evidenciando líquido e proliferação sinovial (setas) na bainha do extensor ulnar do carpo (T). Posteriormente, observam-se as corticais ósseas da ulna distal $(U)$ e do piramidal $(P)$.

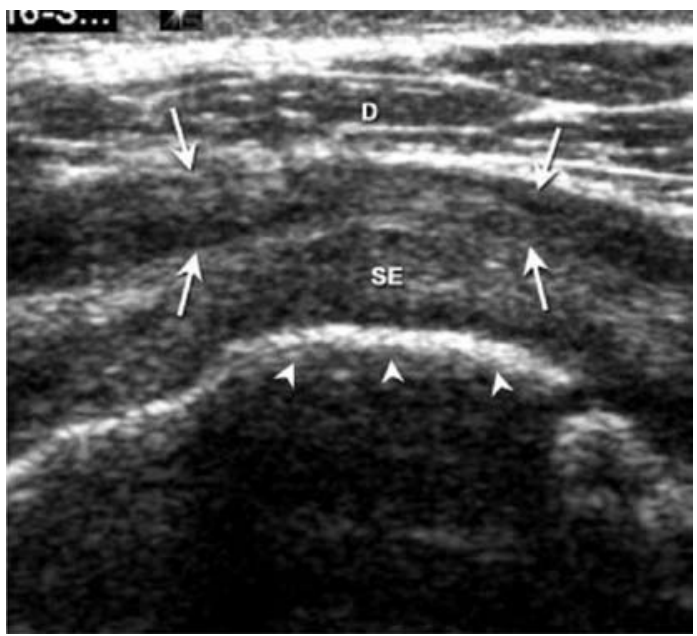

Figura 6 - Bursite. Corte transversal da região anterior da cabeça umeral evidenciando a bolsa subacromial subdeltóidea (setas) com líquido e material levemente hiperecóico que corresponde à proliferação sinovial. Anteriormente, nota-se o músculo deltóide (D) e posteriormente à bolsa, notam-se o tendão do músculo supra-espinal (SE) e a cortical óssea da cabeça umeral (cabeças de setas). 


\section{ULTRA-SONOGRAFIA COMO FATOR PROGNÓSTICO}

$\mathrm{Na}$ ausência de sinovite, erosões não ocorrem. A sinovite subclínica detectada ultra-sonograficamente, isto é, a artropatia inflamatória que não é observada ao exame clínico e detectada ao exame ultra-sonográfico, tem mostrado ser um indicador de mau prognóstico e é mais comum em pacientes com fator reumatóide positivo ${ }^{(33,34)}$. Em um estudo randomizado e controlado com pacientes em terapia anti-TNF-alfa na AR precoce, a ultra-sonografia de base determinou espessamento sinovial e grau de vascularidade nas articulações metacarpo-falângicas e comparou-se com um grupo placebo. Após 18 semanas, os pacientes que receberam anti-TNF-alfa e metotrexato apresentaram redução do espessamento sinovial ao ultra-som, e os pacientes que receberam placebo e metotrexato não demonstraram redução no espessamento sinovial. A evolução radiográfica em 54 semanas indicou não haver progressão das lesões no grupo que recebeu terapia biológica. O retardo ou a reversão dos mecanismos inflamatórios e destrutivos articulares na artrite reumatóide precoce foram aparentes ao ultra-som em 18 semanas e refletiram as alterações radiográficas em 54 semanas $^{(35)}$.

Algumas outras observações indiretas do valor preditivo da ultra-sonografia são dadas pela alta concordância com achados de ressonância magnética, nas quais sinovite, edema ósseo e erosões ósseas têm mostrado valor preditivo em relação ao subseqüente dano ósseo destrutivo. Em vários estudos, entretanto, o mais forte indicador na ressonância magnética de futuros danos erosivos é a presença de edema ósseo, que não pode ser visualizado ao ultra-som, portanto, o valor prognóstico da ressonância magnética não pode ser diretamente transferido ao ultra-som ${ }^{(15)}$.

\section{UTILIZAÇÃO NO TRATAMENTO}

O ultra-som pode ser utilizado para guiar procedimentos diagnósticos, como biópsia da membrana sinovial e do córtex ósseo e para injeção de medicamentos intra-articulares ou nas bainhas sinoviais dos tendões. Estudos têm demonstrado uma redução dos marcadores ultra-sonográficos de inflamação sinovial, incluindo parâmetros de escala de cinza e Doppler de amplitude, acompanhando a resposta ao tratamento com vários agentes farmacológicos ${ }^{(32,36)} \mathrm{e}$ refletindo alterações similares nas medidas clínicas e laboratoriais de atividade da doença ${ }^{(16)}$. O ultra-som pode verificar a extensão da doença, avaliar várias articulações e detectar lesões subclínicas ${ }^{(6,12)}$.

\section{CONCLUSÃO}

A grande importância do uso do ultra-som na artrite reumatóide é o acesso objetivo da extensão e da severidade da doença. Estudos de longo prazo são necessários para mostrar melhora quantitativa e qualitativa no seguimento de pacientes por meio da imagem. O ultra-som em pacientes com suspeita de AR não substitui o estudo radiográfico para verificar dano ósseo precoce. Pode ser utilizado como ferramenta complementar nos pacientes com alto risco de artropatia inflamatória, nos quais as radiografias são normais ou para reexaminar lesões indeterminadas detectadas nas radiografias ${ }^{(6)}$.

Dentre os achados ecográficos, a proliferação sinovial é a ocorrência mais precoce.

Sinais de vascularização no Doppler de amplitude podem facilitar a diferenciação entre tecido sinovial ativo e inativo.

Há dificuldades em se fazer diagnóstico de AR em seus estágios iniciais, e o principal problema é o fato de seu aspecto mais definitivo ser a cronicidade da doença. Os critérios de classificação para AR antes de 12 semanas de evolução da doença não são satisfatórios e, por esse fato, na suspeita de doença articular inflamatória, para determinar a presença de sinovite subclínica, tenossinovite, entesite ou erosões ósseas, o estudo ultra-sonográfico pode ser utilizado $^{(15)}$. Para se realizar o tratamento precoce, estudos de imagem terão implicações na definição de futuros critérios diagnósticos ${ }^{(34)}$.

Outros achados ultra-sonográficos, como derrame articular, erosões ósseas, tenossinovites e bursites, podem ser caracterizados na evolução da patologia.

O exame ultra-sonográfico também parece ser útil para o monitoramento da eficácia terapêutica, demonstrando sinais de diminuição da atividade inflamatória após o início do tratamento. Finalmente, o ultra-som possibilita a biópsia de tecido sinovial, além de orientar a aplicação de medicamentos.

A evolução dos equipamentos de ultra-som, profissionais, bem capacitados e o atual conhecimento da patologia permitem o diagnóstico de alterações subclínicas, a instituição de tratamento precoce e seu monitoramento, na tentativa de evitarem-se as deformidades conseqüentes do curso evolutivo da AR. 


\section{REFERÊNCIAS}

1. Sommer OJ, Kladosek A, Weiler V, Czembirek H, Boeck M, Stiskal M: Rheumatoid arthritis: a practical guide to state-ofart imaging, image interpretation, and clinical implications. Radiographics 25: 381-98, 2005.

2. Rindfleisch A, Muller D: Diagnosis and management of rheumatoid arthritis. Am Fam Physician 72: 1037-47, 2005.

3. Harris Jr ED, Shur PH: Risck factors for possible causes of rheumatoid arthritis. Up to Date 14: 1, 2006.

4. Hau M, Schultz H, Tony HP, et al.: Evaluation of pannus and vascularization of the metacarpophalangeal an proximal interphalangeal joints in rheumatoid arthritis by High-Resolution Ultrasound (Multidimensional Linear Array). Arthritis Rheum 42: 2303-8, 1999.

5. Firestein GF: Evolving concepts of rheumatoid arthritis. Nature 423: 356-61, 2003

6. Wakefield RJ, Kong KO, Conaghan PG, Brown AK, O'Connor PJ, Emery P: The role of ultrasonography and magnetic resonance imaging in early rheumatoid arthritis. Clin Exp Rheumatol 21 (suppl 31): S42-9, 2003.

7. Weyand CM: Immunopathologic aspects of rheumatoid arthritis: who is the conductor and who plays the immunologic instrument? J Rheumatol 34(79): 9-14, 2007.

8. Szkudlarek M, Court-Payen M, Strandberg C, Klarlund M, Klausen T, Ostergaard M: Power Doppler ultrasonography for assessment of synovitis in the metacarpophalangeal joints of pacients with rheumatoid arthritis. Arthritis Rheum 44: 2018-20, 2001.

9. Wakefield RJ, Brown AK, O'Connor PJ, Emery P: Power Doppler sonography: improving disease activity assessment in inflamatory musculoskeletal disease. Arthritis Rheum 48: 285-8, 2003.

10. Grassi W: Clinical evaluation versus ultrasonography: who is the winner? J Rheumatol 30: 5, 2003.

11. Farrant JM, O'Connor PJ, Grainger AJ: Advanced imaging in rheumatoid arthritis. Part 1: Synovitis. Skeletal Radiol 36: 269-79, 2007.

12. Wakefield RJ, Green MJ, Marzo-Ortega H, et al.: Should oligoarthritis be reclassified? Ultrasound reveals a high prevalence of subclinical disease. Ann Rheum Dis 63: 382-5, 2004.

13. Szkudlarek M, Court-Payen M, Jacobsen S, Klarlund M, Thomsen HS, Ostergaard M: Interobserver agreement in ultrasonography of the finger and toe joints in rheumatoid arthritis. Arthritis Rheum 48: 955-62, 2003.

14. Szkudlarek M, Narvestad E, Klarlund M, Court-Payen M, Thomsen HS, Ostergaard M: Ultrasonography of the metatarsophalangeal joints in rheumatoid arthritis: comparison with magnetic resonance imaging, conventional radiography, and clinical examination. Arthritis Rheum 50: 2103-12, 2004.

15. Ostergaard M, Ejbjerg B, Szkudlarek M: Imaging in early rheumatoid arthritis: roles of magnectic resonance imaging, ultrasonography, conventional radiography and computed tomography. Best Pract Res Clin Rheumatol 19: 91-116, 2005.

16. Brown AK, Wakefield RJ, Conaghan PG, Karim Z, O’Connor PJ, Emery P: New approaches to imaging early inflammatory arthritis. Clin Exp Rheumatol 22(Supl. 35): S18-25, 2004.

17. Wakefield RJ, Karim Z, Conaghan PG, et al.: Sonography is more sensitive than clinical examination at detecting synovitis in the metatarsophalangeal joints than clinical examination. Arthritis Rheum 42: S352, 1999.
18. Ostergaard MO, Wiell C: Ultrasonography in rheumatoid arthritis: a very promising method still needing more validation. Curr Opin Rheumatol 16: 223-30, 2004.

19. Backaus M, Kamradt T, Sandrock D, et al.: Arthrits of the finger joints. Arthritis Rheum 42: 1232-45, 1999.

20. Keen HI, Emery P: How should we manage early rheumatoid arthritis? From imaging to intervention. Curr Opin Rheumatol 17: 280-5, 2005.

21. Hermann KG, Backhaus M, Schneider U, et al.: Rheumatoid arthritis of the shoulder joint. Comparison of conventional radiography, ultrasound, and dynamic contrast-enhancend magnetic resonance imaging. Arthritis Rheum 48: 3338-49, 2003.

22. Joshua F, Lassere M, Bruyn GA, et al.: Summary findings of a systematic review of the ultrasound assessment of synovitis. J Rheumatol 34: 839-47, 2007.

23. Conaghan PG: Musculoskeletal ultrasonography: Improving our senses. Arthritis Rheum 53: 639-42, 2005.

24. Brasseur JL: Advances in osteoarticular ultrasonography. Joint Bone Spine 68: 6-9, 2001.

25. Taylor PC: VEGF and imaging of vessels in rheumatoid arthritis. Arthritis Res (suppl 3): S99-107, 2002.

26. Terslev L, Torp-Pedersen S, Savnik A, et al.: Doppler ultrasound and magnetic resonance imaging of synovial inflammation of the hand in rheumatoid arthritis. Arthritis Rheum 48: 2434-41, 2003.

27. Walther M, Harms H, Krenn V, Radke S, Faehndrich TP, Gohlke F: Correlation of power Doppler sonography with vascularity of the synovial tissue of the knee joint in patients with osteoarthritis and rheumatoid arthritis. Arthritis Rheum 44: 331-8, 2001.

28. Newman JS, Laing TJ, McCarthy CJ, Adler RS: Power Doppler sonography of synovitis: Assessment of therapeutic response Preliminary observations. Radiology 198: 582-4, 1996.

29. Klauser A, Frauscher F, Schirmer M, et al.: The value of contrast-enhanced color Doppler ultrasound in the detection of vascularization of finger joints in patients with rheumatoid arthritis. Arthritis Rheum 46: 647-53, 2002.

30. Farrant JM, O'Connor PJ, Grainger AJ: Advanced imaging in rheumatoid arthritis. Part 2: Erosions. Skeletal Radiol 36: 381-9, 2007.

31. Lopez-Ben R, Bernreuter WK, Moreland LW, Alarcon GS: Ultrasound detection of bone erosions in rheumatoid arthritis: a comparison to routine radiographs of the hands and feet. Skeletal Radiology 33: 80-4, 2004.

32. Heijde D: Impact of imaging in established rheumatoid arthritis. Best practise and research. Clin Rheumatol 17: 783-90, 2003.

33. Bresnihan B, Kane D: Sonography in subclinic synovitis. Ann Rheum Dis 63: 333-4, 2004.

34. Quinn MA, Green MJ, Conaghan P, Emery P: How do you diagnose rheumatoid arthritis early? Best Practise and Research Clin Rheumatol 15: 49-66, 2001.

35. Taylor PC, Steuer A, Gruber J, et al.: Comparison of ultrasonographic assessment of synovitis and joint vascularity with radiographic evaluation in a randomized, placebo-controlled study of infliximab therapy in early rheumatoid arthritis. Arthritis Rheum 50: 1107-16, 2004.

36. Iagnocco A, Perella C, Ceccarelli E, Tripodo C, Magrini L, Valesini G: Ultrasonographic assessment of the response to Etanercept treatment in patients with rheumatoid arthritis. Reumatismo 58(3): 233-8, 2006. 